\title{
THE INTEGRATION OF TRADITIONAL AND SUSTAINABLE ARCHITECTURE AS AN INPUT TO SOLVE THE PROBLEM OF ENERGY SAVING IN MODERN URBAN COMMUNITIES
}

\author{
'and Emad Fahim matta ${ }^{2}$ Sally Hosny Alkholy ${ }^{1}$ \\ ${ }^{1}$ Architecture Engineer in New Cairo Authority \\ ${ }^{2}$ Architecture in Helwan University - Mataria branch \\ sally_20081@yahoo.com, emad.kamel@ark-eg.com
}

\begin{abstract}
The progress and technological growth led to increasing the human needs to energy and thus increase the general consumption of energy in the building, all this in light of the energy crisis facing the world as a result of the almost total reliance on non-renewable sources of energy and the clear trend towards respect for the environment and its protection from pollution. To the use of renewable energy sources was the best solution for all these problems and so many of the modern architectural trends began to use renewable energy systems in the building and even became important decisions taken by the architect through different stages of design and Therefore, its use in the building should affect the design and shape of the building.

The rich experience of traditional Arab architecture and its architectural vocabulary and natural materials are combined with modern trends of sustainable architecture to reach an integrated architectural model that combines the advantages and benefits of both directions. The building benefits from the tremendous development of technology and computer, as well as the revival of traditional architecture and derived from a rich and integrated life experience.
\end{abstract}

Key words : Traditional Architecture - Sustainable Architecture - Solve The Problem Of Energy In Bulding- Saving Energy In Modern Buildings .

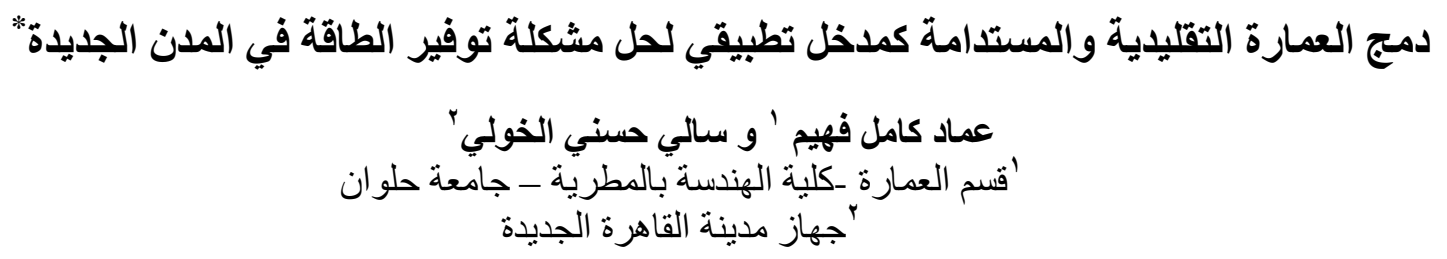


الكلمات الدالة : العمارة التقليدية - العمارة المستدامة - المباني الحديثة - توفير الطاقة _ الهوية المعمارية -

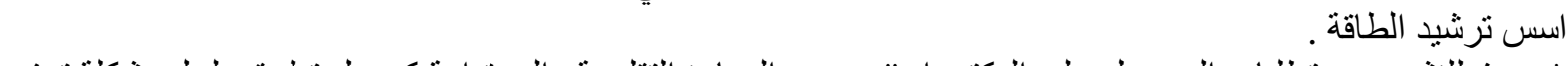

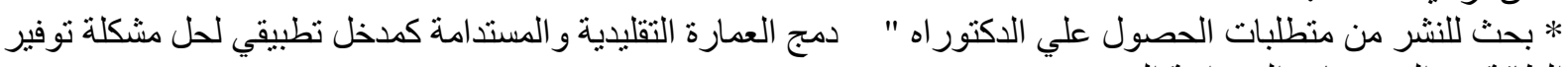
الطاقة في المجتمعات العمر انبة الجديدة".

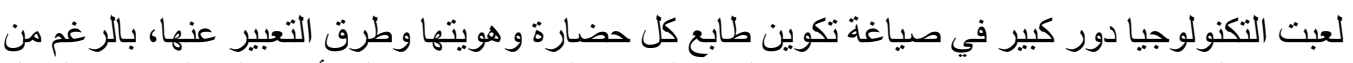
تقايم :

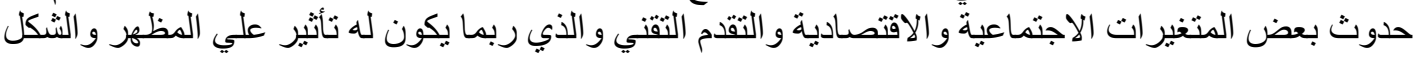

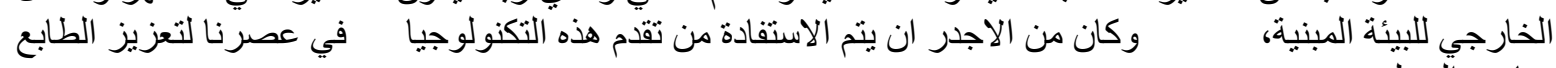

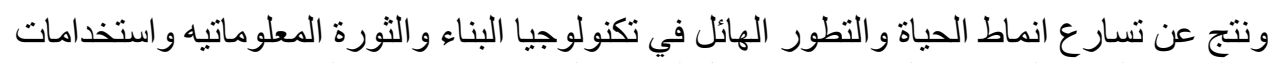

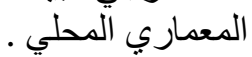

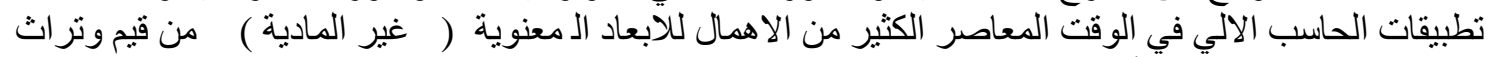

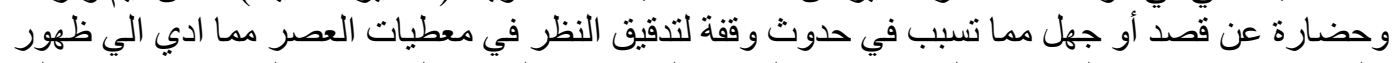

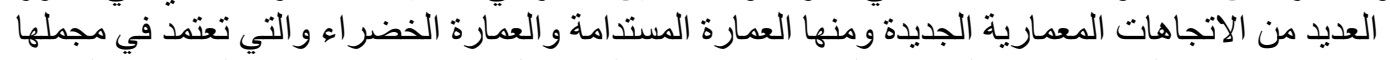

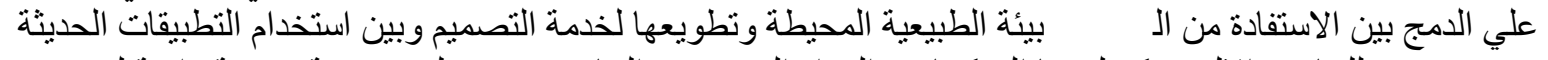
للحاسب الالي ونكنولوجيا التركيبات و المو اد الجديدة في البناء مجتمعين لتوفير بيئة مريحة و امنة لمستخدم

المبني.

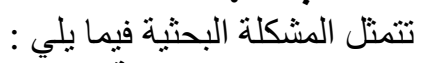

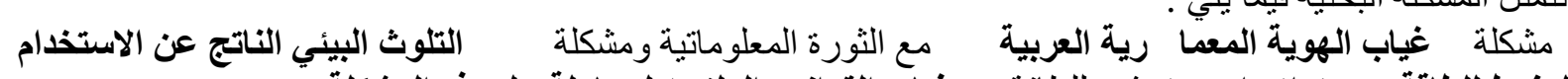

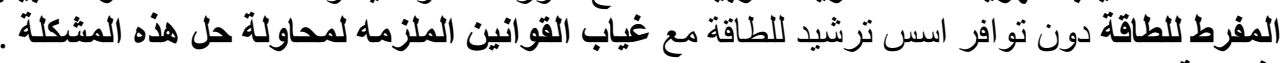
فرضية البحث : مفرطة

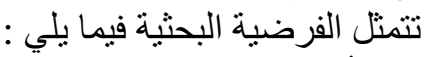
أن تطبيق الدمج بين مفردات العيمة العمارة العربية التقليدية و العمارة المستدامة سيؤدي الي ترشيد وتوفير الطاقة في

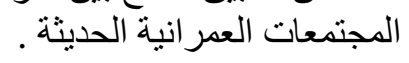
الهدف من البحث :

الوصولّ الي مبني متكامل يجمع بين الحفاظ علي هوية ل

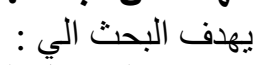

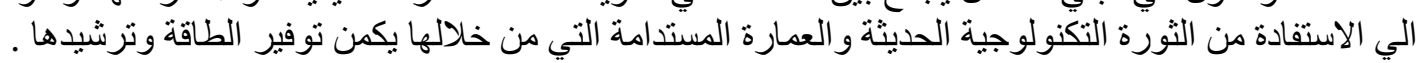

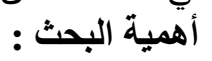
أهمية ألتعرف على تطبيق العمارة العمارة المستدامة في المباني لترشيد الطاقة وكيفية الاستفادة من العمارة التقليدية

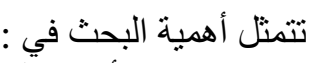

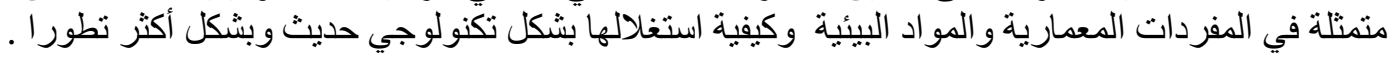

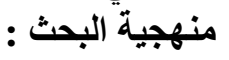

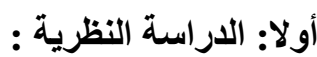

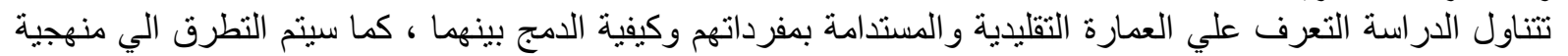

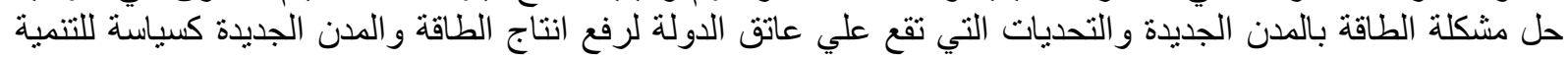

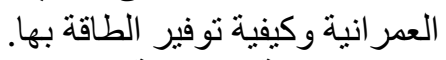
ثانيا: الاراسة التطبيقية : مانية تتناول الدراسة التطبيقية تحليل مبني موفر للطاقة من خلال المعالجات البيئية المستخدمة به من خلال استخدام

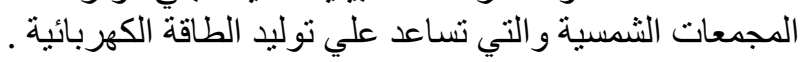

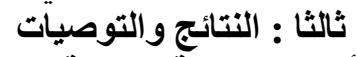

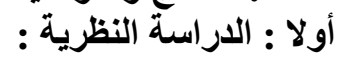

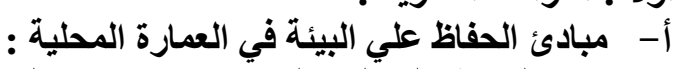

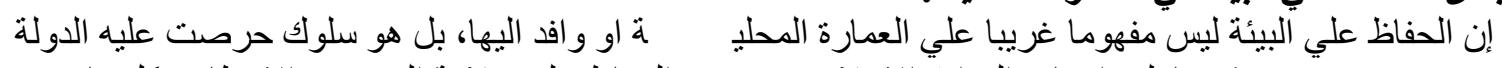

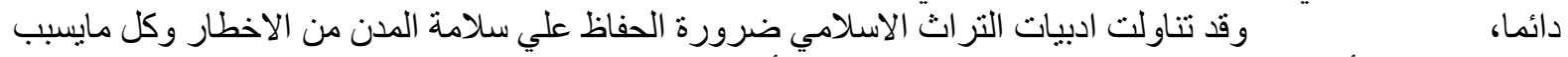

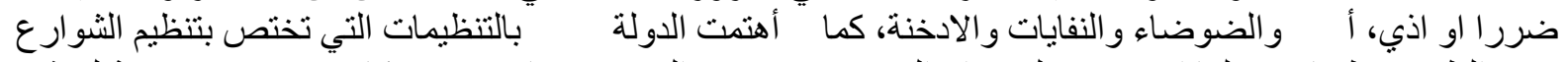

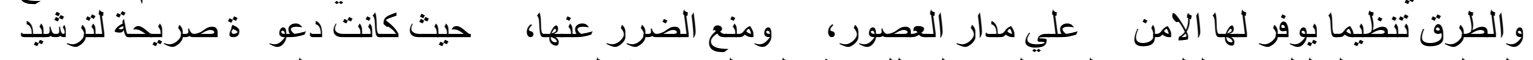

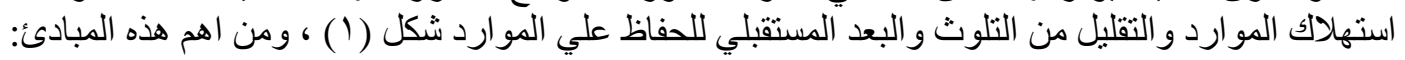




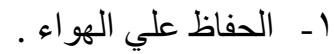

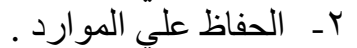

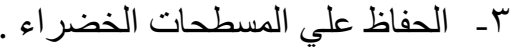

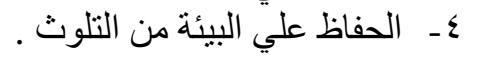

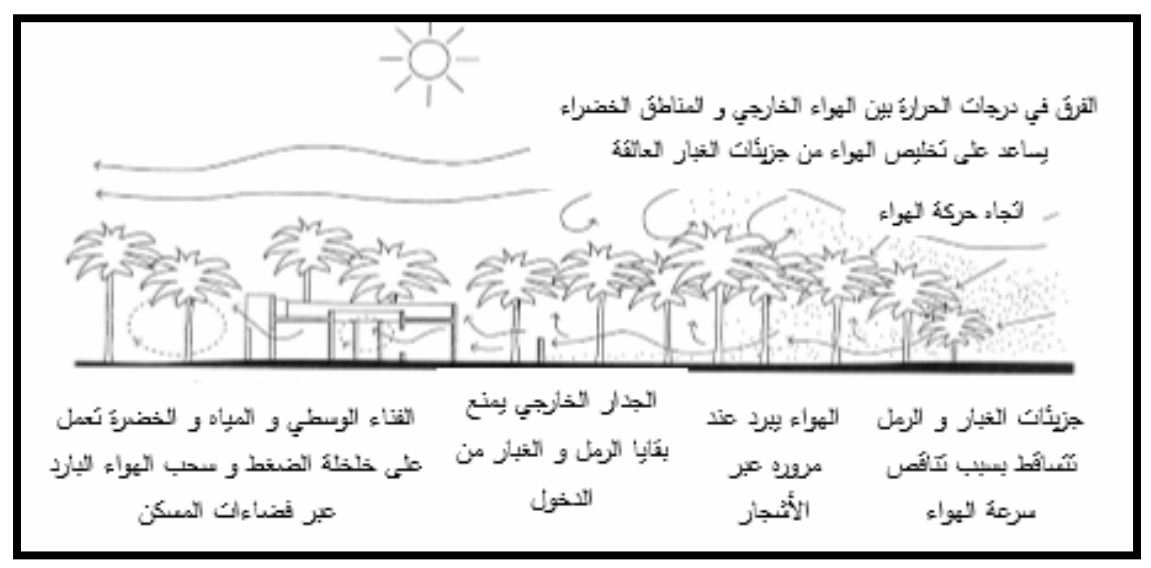

شكل (1) الحفاظ علي البيئة في العمارة المحلية من خلال الحفاظ علي الهواء ، المسطحات الخضراء، الحفاظ علي البيئة من التلوث

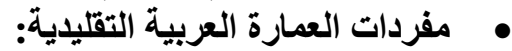

تمثل العمارة المحلية التقليدية جميع موروثات الثعوبة التعابة العربية من المباني ذات الطابع المميز و التي

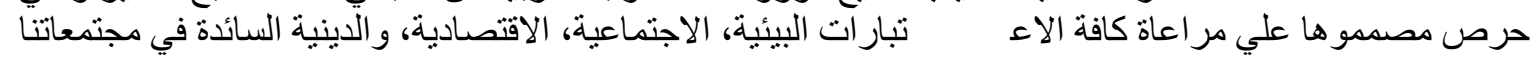

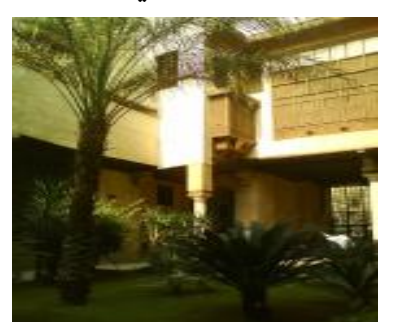

الفناء الداخلي هو احد السمات المعمارية و الرمزية للمسكن في العمارة

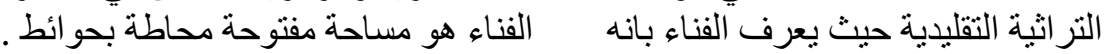

شكل r يوضح الأفنية في المسكن التقليدي

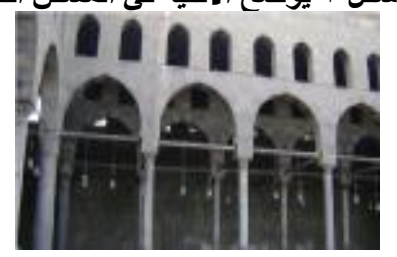

• ت العقود : ت العقود من العناصر المعمارية الهامة في العمارة التقليدية فهي تقوم

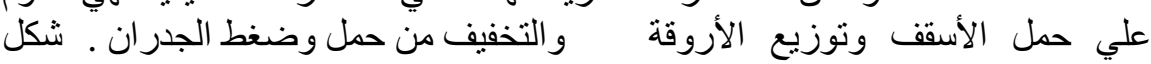

شكل ب يوضح شكل العقود

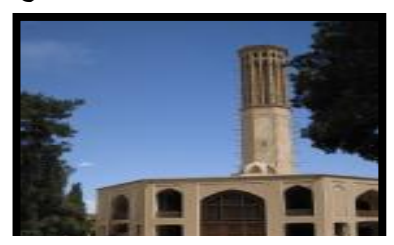

شكل ؛ يوضح شكل الملاقف

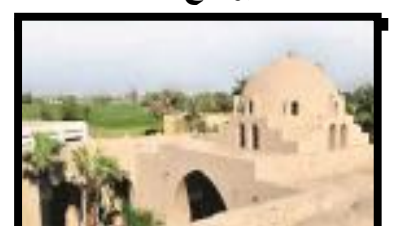

شكل • ميوضح شكل القباب

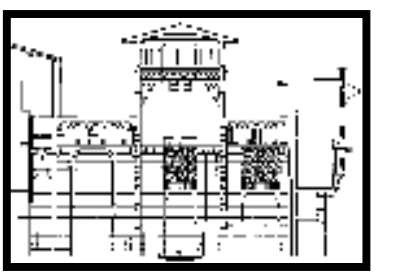

JAUES, 14, 52, 2019
• الملاقف :

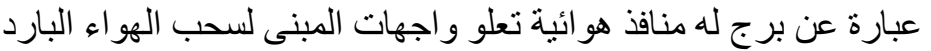

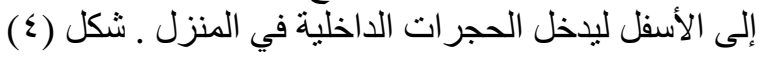

$$
\text { • القباب والاقبية : }
$$

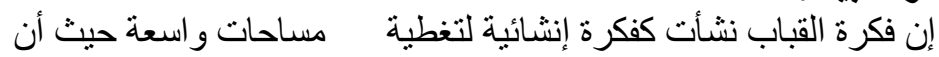

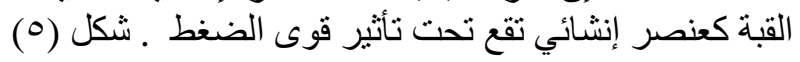

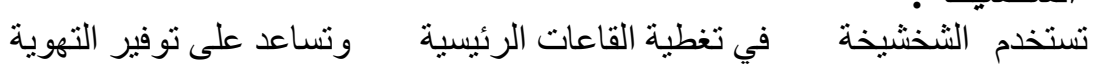

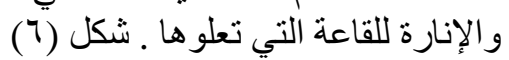

شكل 1 يوضح شكل الشخشيخة 
هي عبارة عن فتحات داخلية شبكية خشبية ذات مقطع دائري تفصل بينها مسافات محددة ومنتظمة

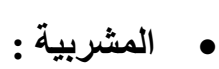

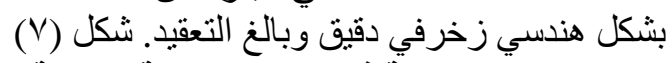

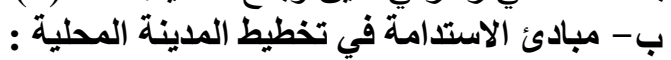

تعتبر المدينة العربية بنسيجها المتضام التقليدي أفض ل مثنال علي تطبيق مفهوم الاستدامة علي مستوي

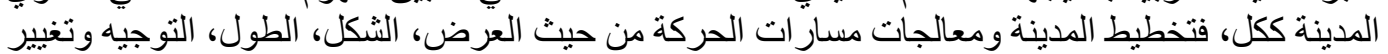

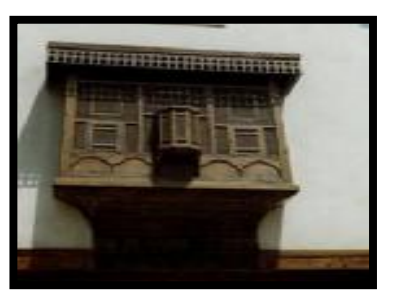

شكل V يوضح شكل المشربية

الاتجاه يمثل المرحلة الاساسية للتكييف مع البيئة .

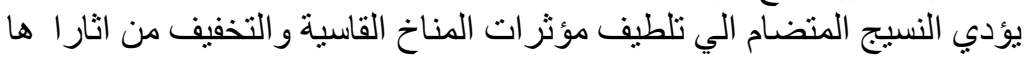

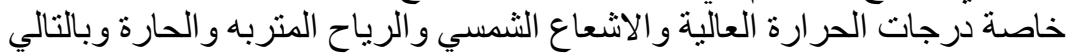

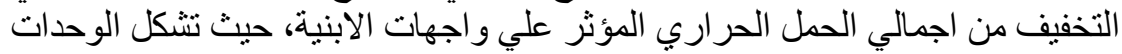

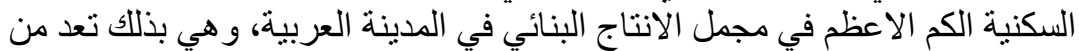

المؤثرات القوية في المعطيات البيئية.

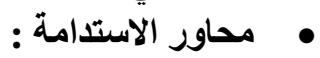

حيث تحتوي العمارة المستدامة الي محورين رئيسين تهدف الى تعزيز التتمية

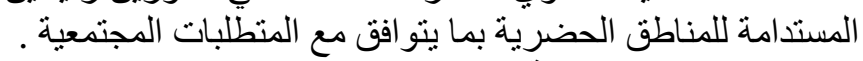

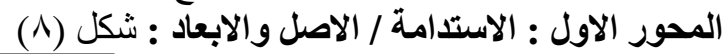

المحور الاول للاسنالمة الاصل و الابعاد

تطبيق الأفكارالالاقتصادية الجديدة

من خلال توفير الطاقات المختلفه

بجميع ادو اتها

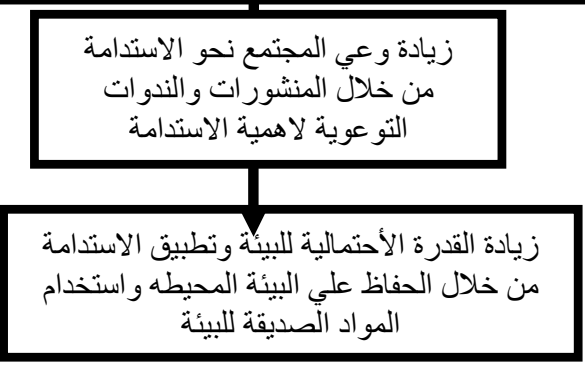

شكل ^ المحور الاول للاستدامه
اساليب مبتكلة في الأقتصاد من خلال التوفير علي المدي الأمدي البعيد للطاقات المختلفة البري

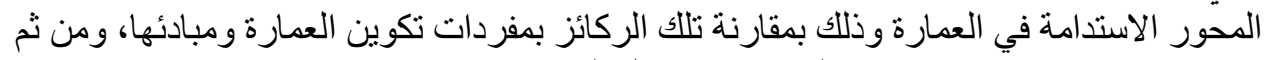

المحور الثاني : الاستدامة في العمارة :

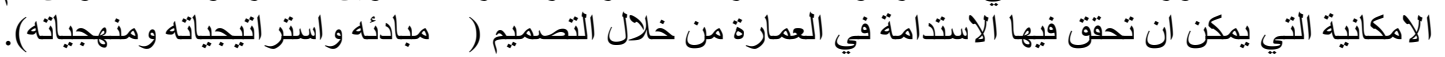

جدول ا اطار المفاهيم للمبني المستدام

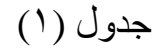

\begin{tabular}{|c|c|c|}
\hline المنهجيات & الأستراتيجيات & المبادئ \\
\hline التقليل من ادخال الموارد غير المتجددة الي المباني المنفايات & الطاقة الماظ علي : الموارد & الأقتصاد في \\
\hline التأثير علي البيئة . & 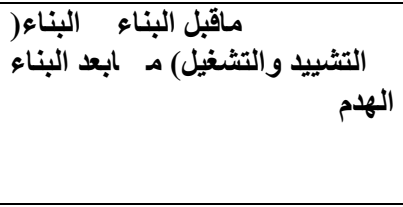 & دورة حياة \\
\hline تحسين نوعية حياة البشر والفئات الأخري & الطبيعية & التصميم \\
\hline
\end{tabular}

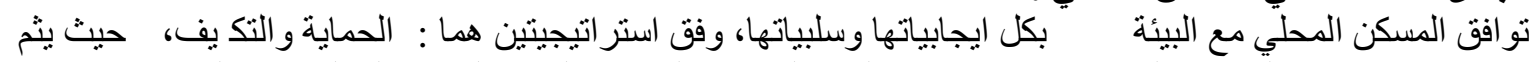

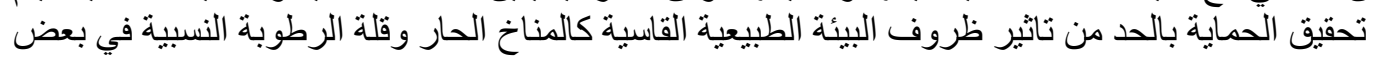

المناطق وارتفاعها في مناطق اخري وشدة الاشعاع الثمسي. 
اما التكيف فكان باستغلال الامكانيات الكامنة لهذه الظروف القاسية و التعامل معها بما يحقق الرا حة الحر ارية

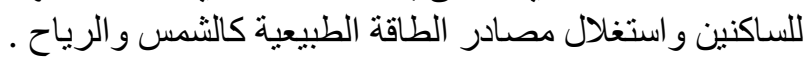

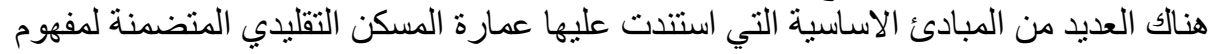

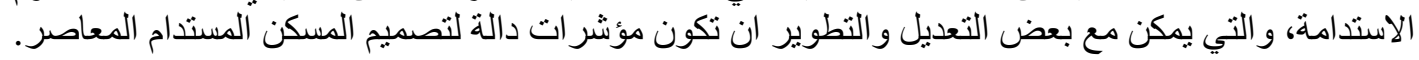

شكل (9) - 19)

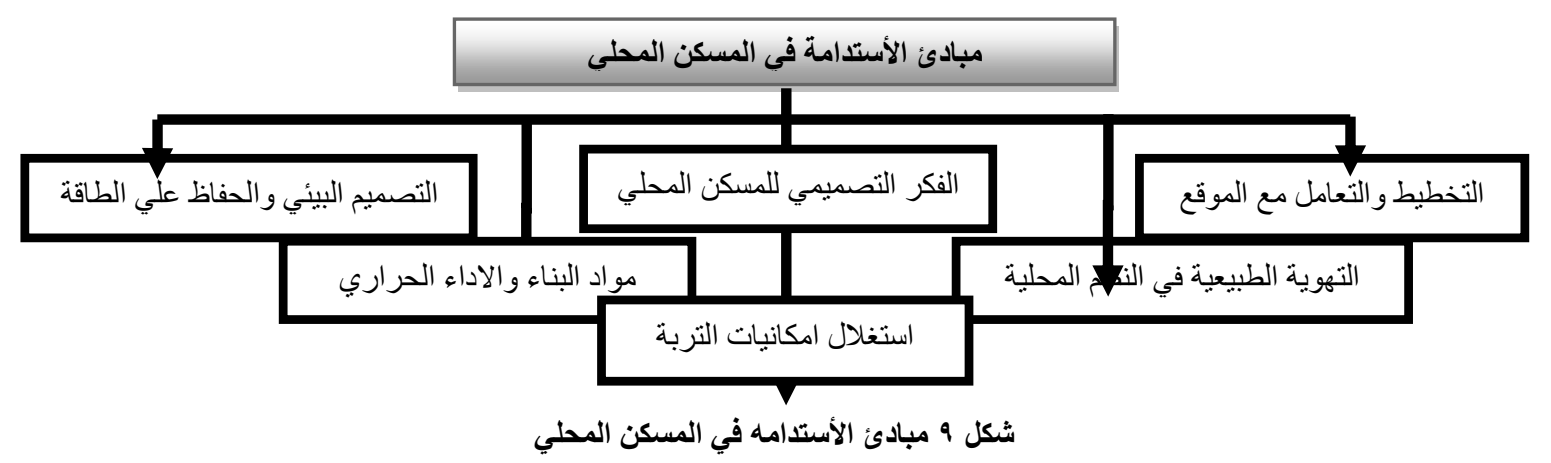

ث- استر اتيجية دمج العمارة المحلية بالعمارة المستدامة : تم استخلاص استر اتيجيات توجهات العمات دمج العمارة المحلية بالعمارة المستدامة حيث سيتم الجمع بينهما

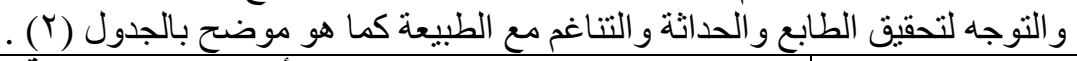

\begin{tabular}{|c|c|}
\hline الأستراتيجيات المتبعة & تالوجهاتة \\
\hline 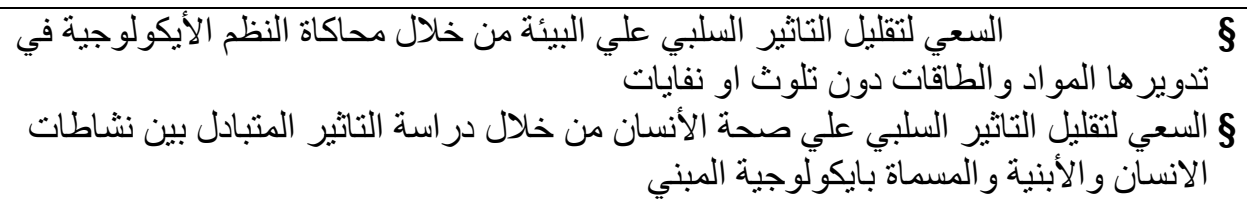 & $\begin{array}{l}\text { مفهوم النظام } \\
\text { (الإيكولوجيف }\end{array}$ \\
\hline 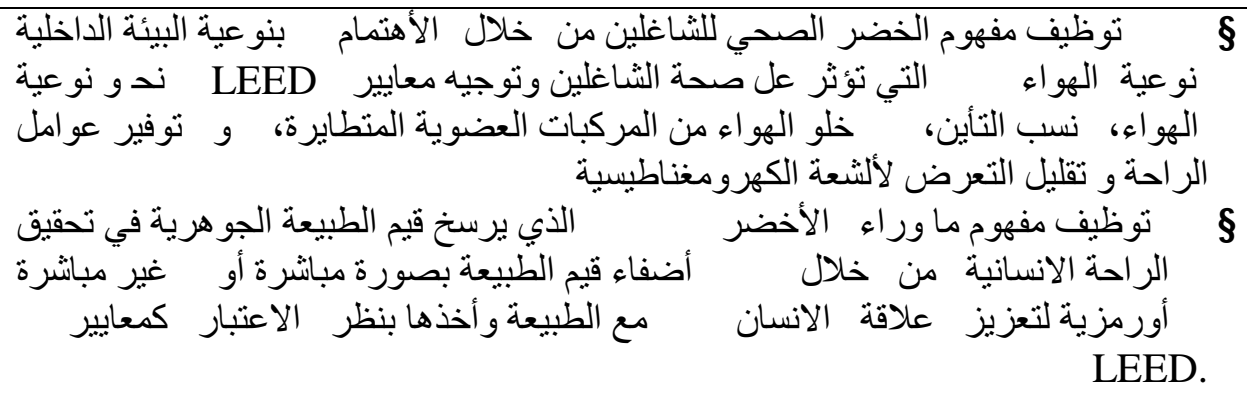 & مفهوم الأخضر) \\
\hline بأقتصاد المو ارد و الطاقات و الحفاظ عليها وتدو ير ها و الحفاظ عل & 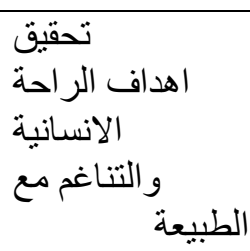 \\
\hline 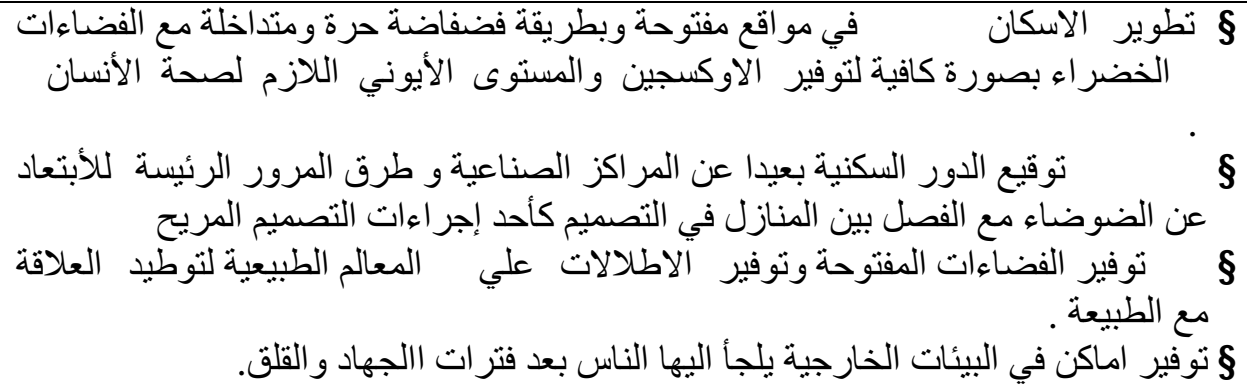 & 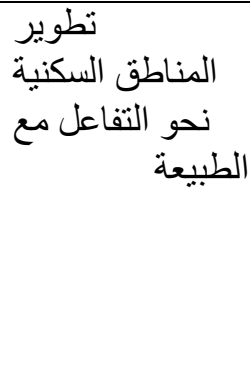 \\
\hline
\end{tabular}




\begin{tabular}{|c|c|}
\hline 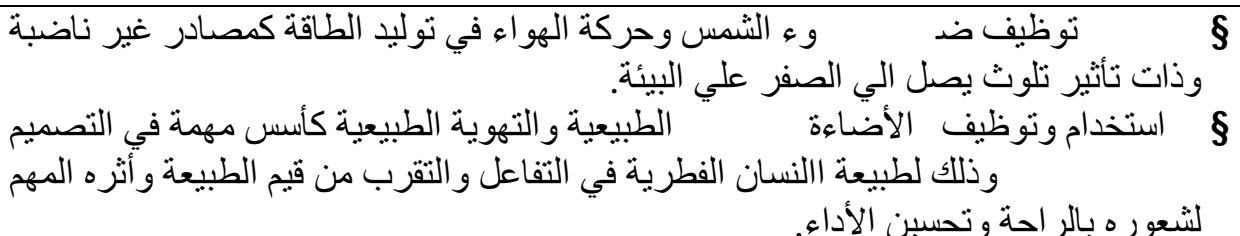 & 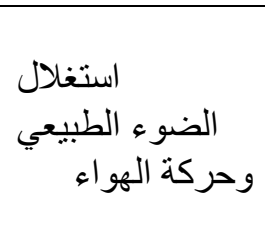 \\
\hline
\end{tabular}

جدول ب إستراتيجية دمج العماءرة العمارة المحلية بالعمارة المستدامة

\section{ج- التحديات التي تقع علي عاتق الدولة لرفع كفاءة انتاج واستهلاك الطاقة بالمدن الجديدة:}

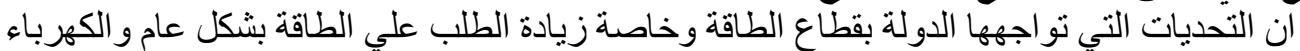

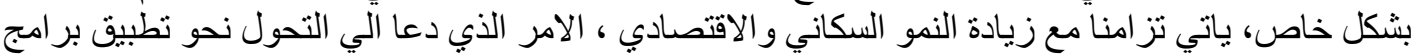

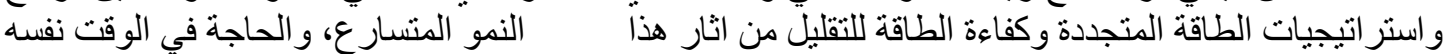

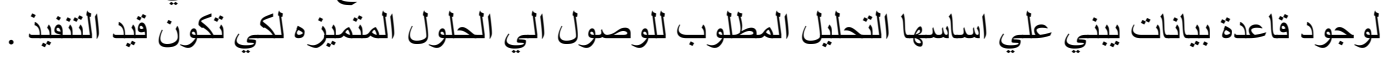

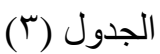

\begin{tabular}{|c|c|}
\hline مجال الطاقة المتجددة وكفاءة الطاقة . للتعرف علي السياسات و البرامج الد عتمده في الدول في & الحلول المتميزة \\
\hline وضع الاستر اتيجيات وبر امج العمل لتعزيز رفع كفاءة و انتاج و استهلاك الطاقة & للتنفيذ لحل مشكلة \\
\hline فيما بينها. محاولة لتبادل الخبرات في مجالات رفع كفاءة وانتاج و استهلاك الطاقة و التطور & الطاقة في الدولة الد \\
\hline
\end{tabular}

\section{جدول ب الحول المتميزة لحل مشكلة الطاقة في الدولة}

\section{ح- منهجية حل مشكلة الطاقة بالمدن الجديدة :}

تتمثل منهجية محاولة حل مشكلة الطاقة بالمدن الجديدة في توفير الطاقة لكافة القطاعات المختلفة بدرجة

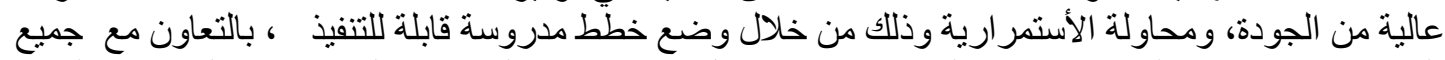

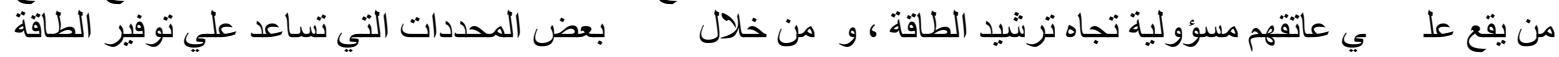

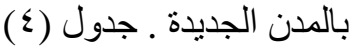

\begin{tabular}{|c|c|}
\hline 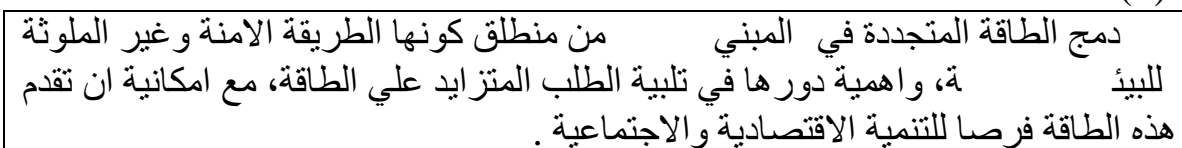 & \\
\hline 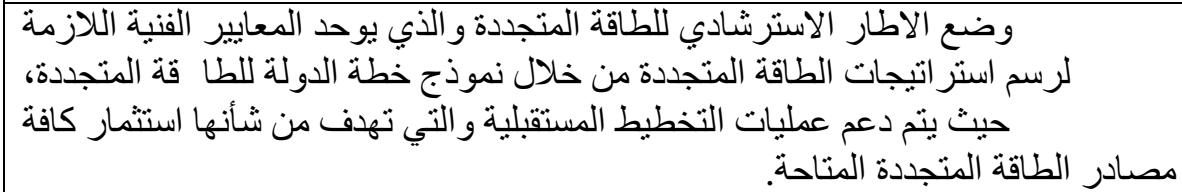 & \\
\hline 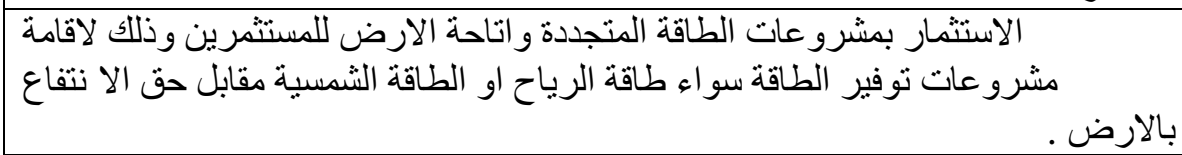 & 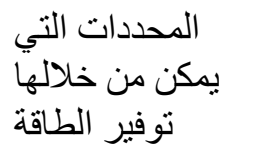 \\
\hline 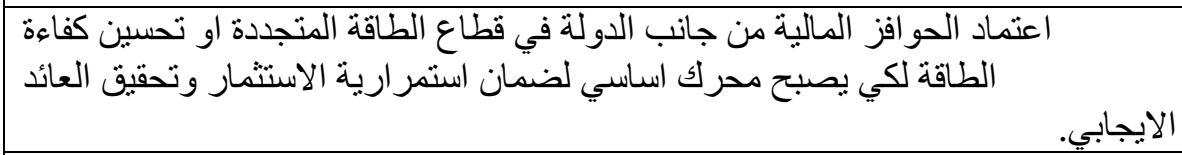 & بالمدن الجديدة \\
\hline 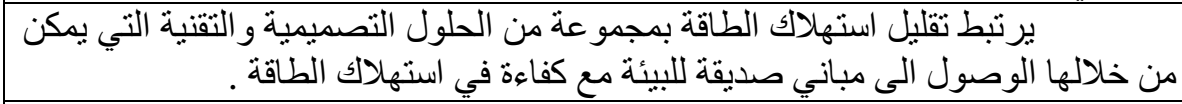 & \\
\hline 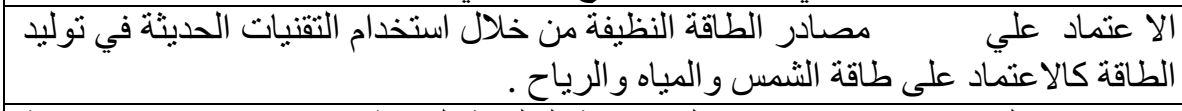 & \\
\hline 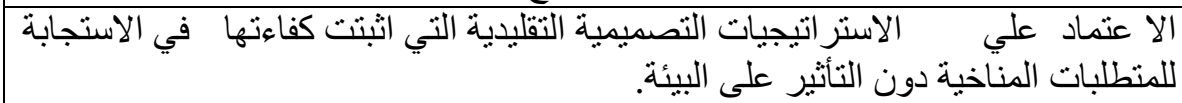 & \\
\hline 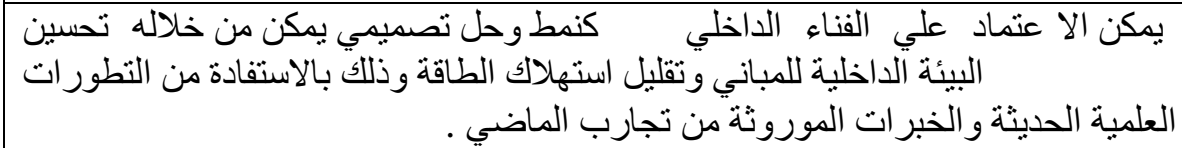 & \\
\hline
\end{tabular}

خ- المدن الجديدة كسياسة للتنمية العمرانية وروئية توفير الطاقة بها:

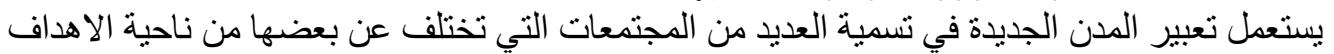

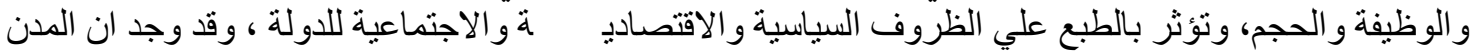


الجديدة تطلب اربعة عناصر اساسية هي : وجود القاعدة الاقتصادية، والاكثفاء الذاتي، و استخدامات الار اضي

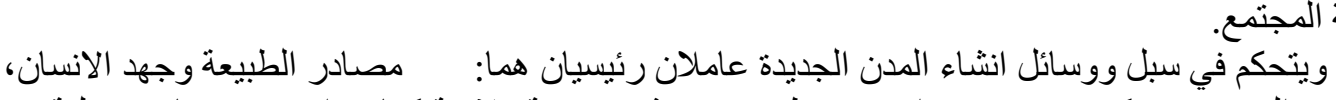

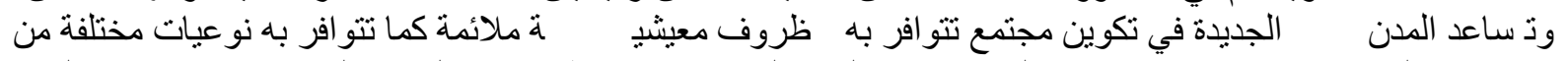

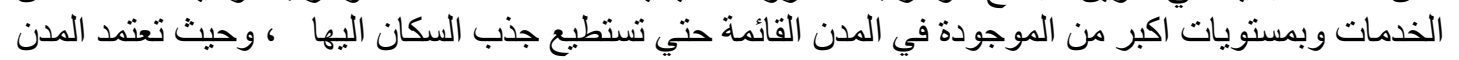

الجديدة علي علاقات مختلفة تؤثر علي انتشار ها شكل (· • (1) .

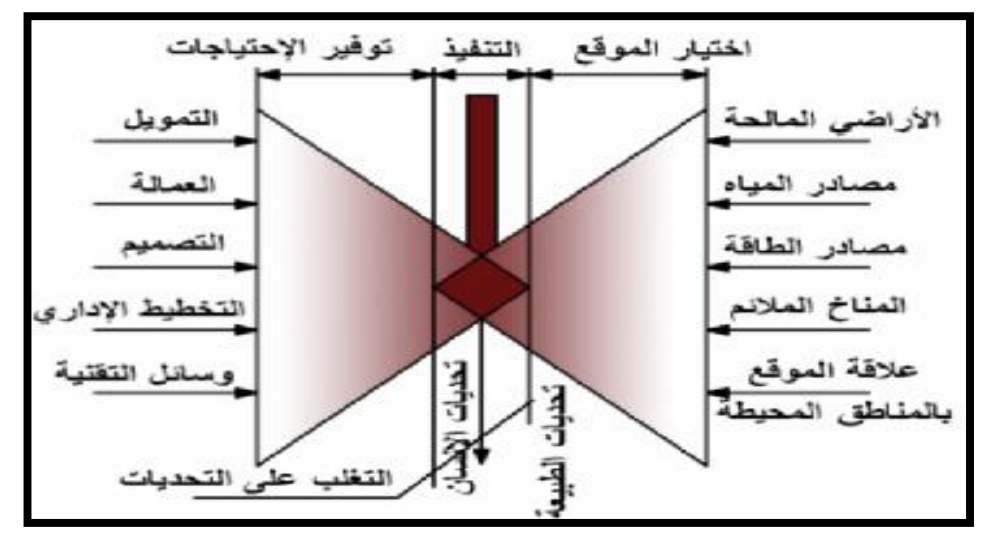

شكل · ل يوضح

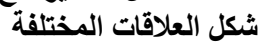

المؤثرة علي انتثار المدينة

الجديدة

تهدف الذر استة التطبيقي

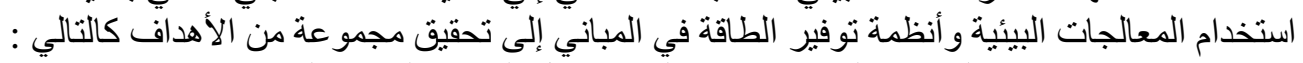

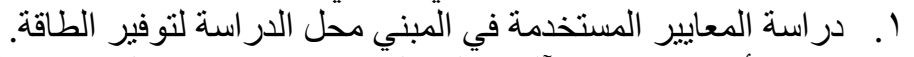

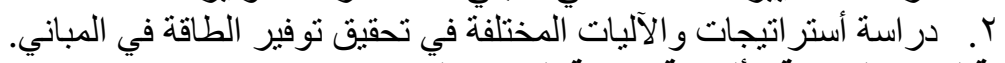

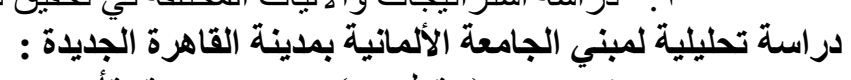

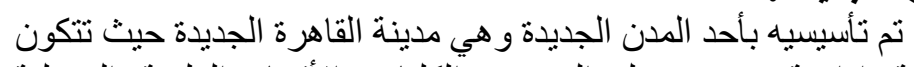

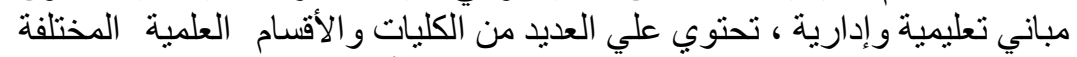

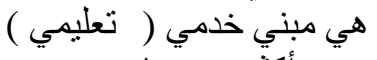

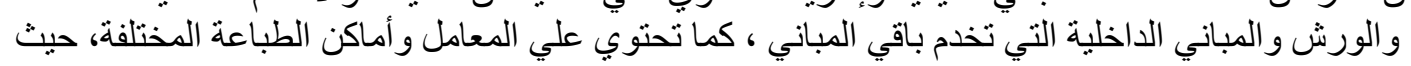

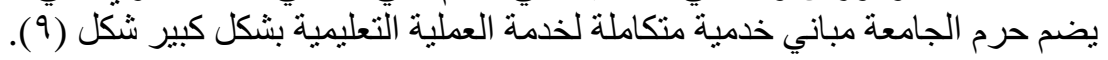

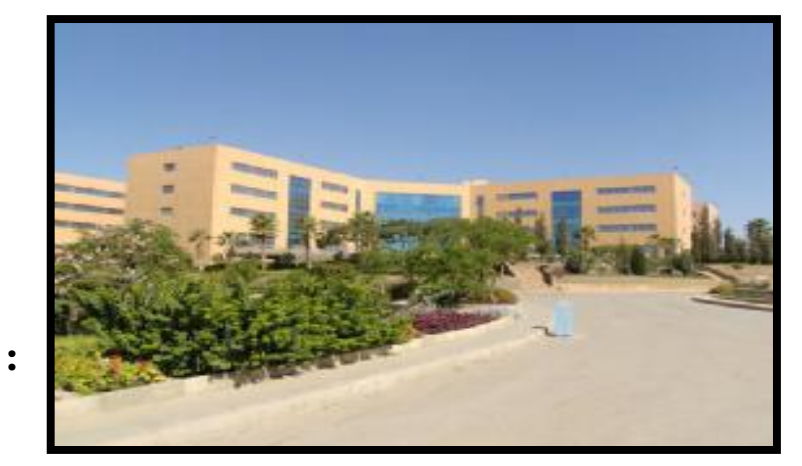

الموقع : مدينة القاهرة الجديدة وصف المشروع :

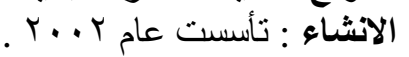

\section{شكل (1 11 ) مبني الجامعة الألمانية}

أستراتيجيات توفير الطاقة المستخدمة بمباني الجامعة

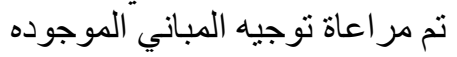

بالجامعة للإستفادة القصوى من الإضـاءة المباءة

$$
\text { و التهوية الطبيعية. }
$$

تُم مر اعاة أيضا استخدام وسائل التظليل للحماية من أثُعة الثمس وتوظيف الأرصفة و الأسقف فاتحة

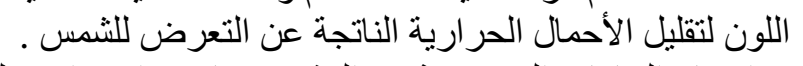

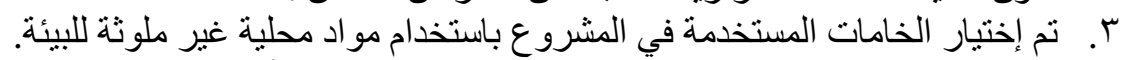

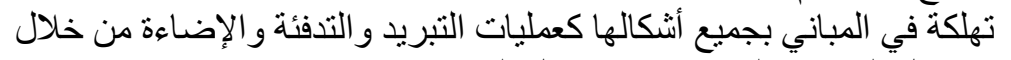

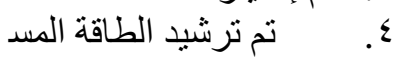

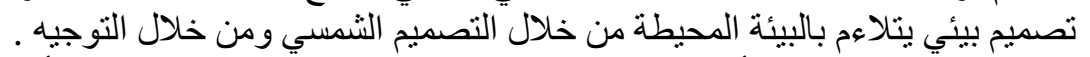

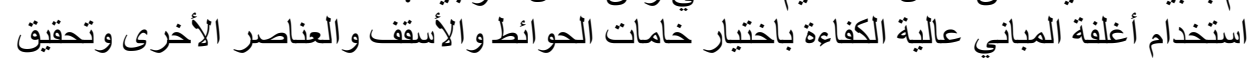
متطلبات العزل و الكفاءة و المنانة .

تحقيق أعلى درجات المرونة و التكيف مع احتياجات مستخدمي مباني الجامعة و أحتياجاتهم الحالية

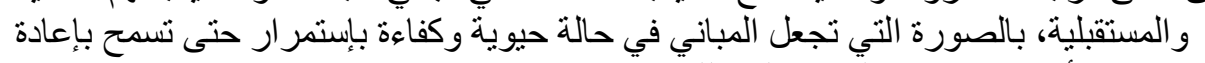

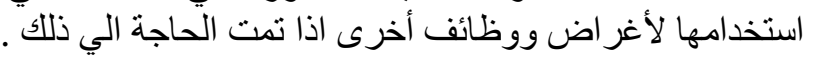

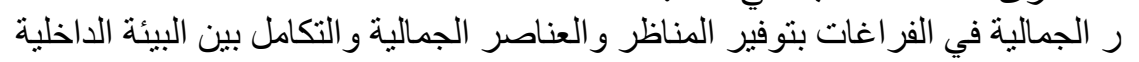

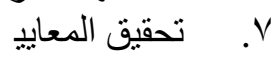

المبنية والبيئة المحيطة . جدول(o) 
جدول (•) توفير الطاقة من خلال الالواح الثمسية في مبني الامتحانات شهريا

\begin{tabular}{|c|c|c|c|}
\hline \multicolumn{4}{|c|}{ توفير الطاقة من خلال الألواح الشمسية في مبني الأمتحانات شهريا } \\
\hline الطاقة الموفرة & الطاقة المستخذمة بعد الالواح & الطاقة المستخدمة قبل الالواح & الكهربائية في المبني الطاقة \\
\hline 97 كيلو وات من الطاقة & $7 \varepsilon$ & 17. & تكييفات وتبريد وتهوية أو \\
\hline 11 الكيلو وات من الطاقة & IT & $\mu$. & 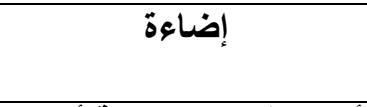 \\
\hline •r الكيلو وات من الطاقية & $r$. & 0. & عالية أحمال قوي كهربائية أخري \\
\hline 7 الكيلو وات من الطاقة & $\varepsilon$ & 1. & أحمال قُوي كهربائية أخري \\
\hline
\end{tabular}

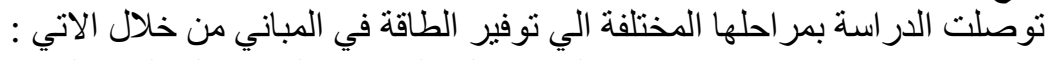

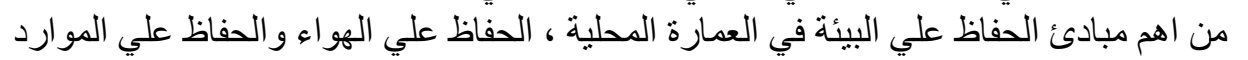

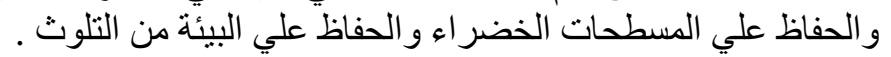

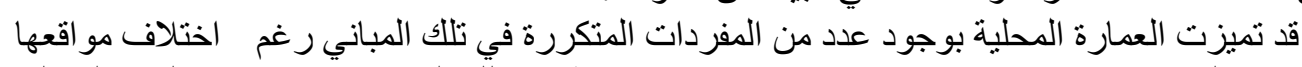

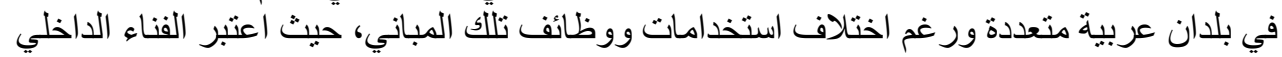

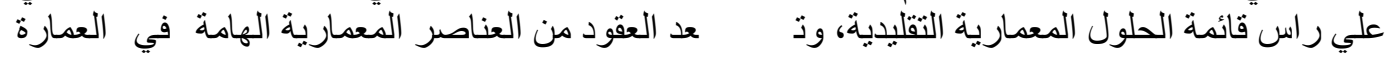

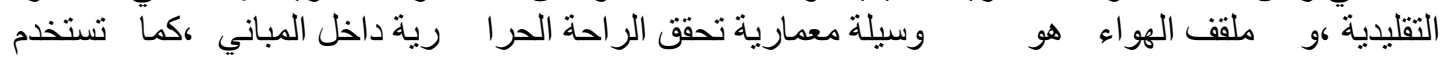

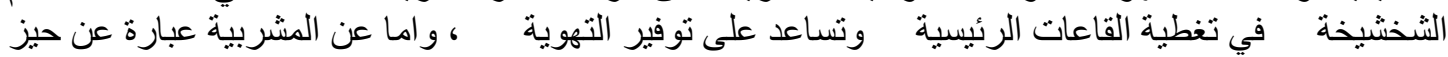

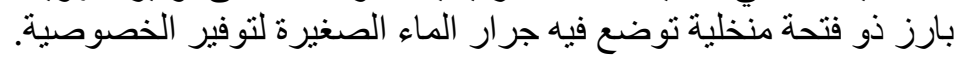

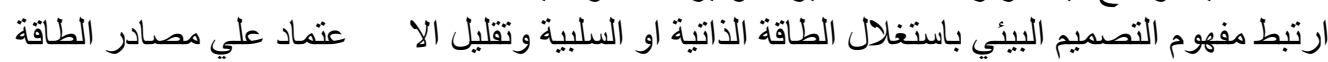

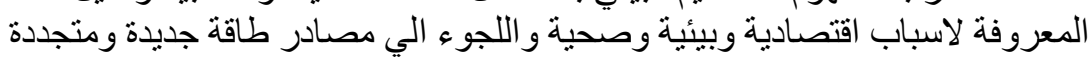

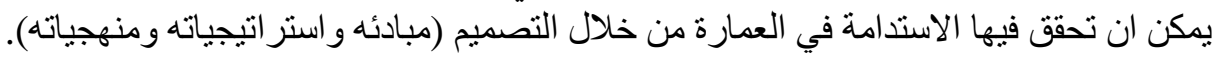

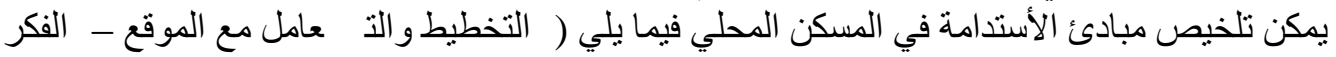

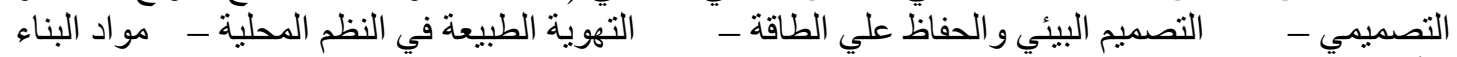

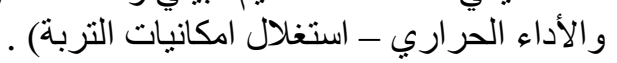

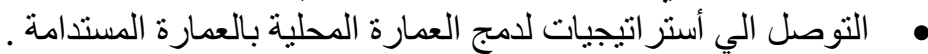

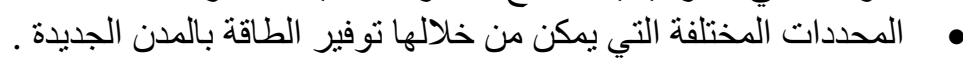

أعتماد العمارة المستدامة ومبادئها واستراتيجياتها كاتجاه تصميمي يستجيب لمتطلبات العمارة المتعددة

رابعا : التوصيات

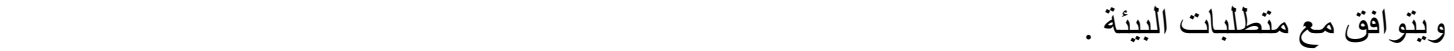

أعنماد مبادئ العمارة المستدامة كمعايير اساسية للبناء وضمن قو انين البناء المع مول بها باعتبار ها من

أهم منطلبات العصر الحديث.

الأخذ بعين الأعتبار المعالجات والأستر اتيجيات التصميمة التقليدية وتطوير ها في المباني المعاصرة

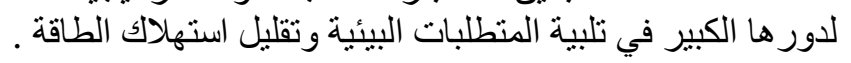

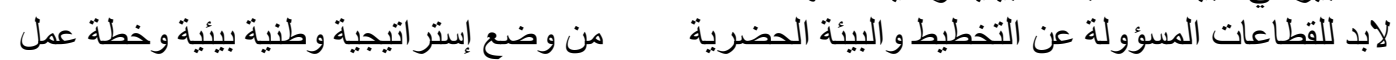

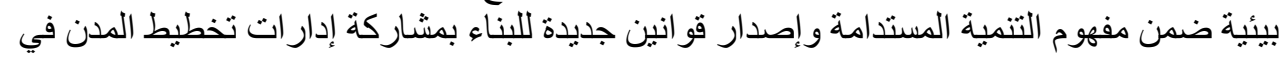

تنفيذ هذه السياسات خلال المرحلة المقبلة .

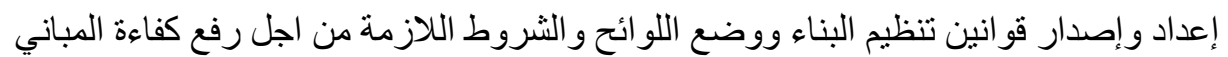

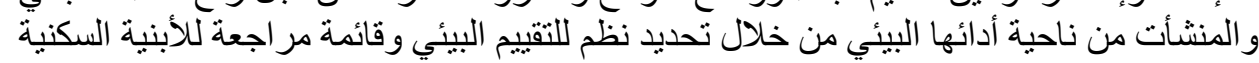

وأنماط الأبنية المتعددة 


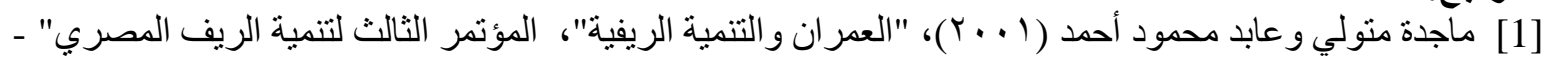
شبين الكوم ـ كلية الهندسة - جامعة المنوفية.

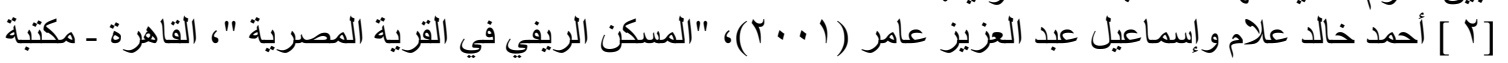

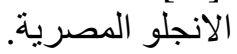

[3] AL-Zubaidi, Mahah S (2002), "Mass-effect Passive Cooling An Environmental Friend Technology, Towards Better Built Environment : Innovation, Sustainability and Technology", Monash Univ, Australia

[4] london.gov.uk/learning/docs/inside_city_hall.pdf

[5] Koones. Sheri (2010), "Prefabulous + Sustainable: Building and Customizing an Affordable, Energy-Efficient Home”, Butterworth Architecture, Landon , (2010).

[6] Gissen, David (2003), "Big \& Green: Toward Sustainable Architecture in the $21^{\text {st }}$ Century", Princeton Architectural, New York, USA.

[7] Technology Roadmap: Energy-efficient Buildings,2011 - Heating and Cooling Equipment, page 5:

[8] Bainbridge. David (2011), "Passive Solar Architecture: Heating, Cooling, Ventilation, Daylighting and More Using Natural Flows ", The Mit Press, New York.

[9] Picon Antoine (2010), "Digital Culture in Architecture", Butterworth Architecture, Landon. 